\title{
Cord blood levels of uric acid, lipid profile and HOMA-IR in pregnancy induced hypertension
}

\author{
Priyadharshini R.*, V. Vijayakumari
}

Department of Biochemistry, ESIC MC and PGIMSR, Bangalore, Karnataka, India

Received: 24 August 2021

Revised: 28 October 2021

Accepted: 28 November 2021

\section{*Correspondence:}

Dr. Priyadharshini R,

E-mail: priyasethu30793@gmail.com

Copyright: (C) the author(s), publisher and licensee Medip Academy. This is an open-access article distributed under the terms of the Creative Commons Attribution Non-Commercial License, which permits unrestricted non-commercial use, distribution, and reproduction in any medium, provided the original work is properly cited.

\section{ABSTRACT}

Background: Cardiovascular diseases (CVD) is a major global health burden that results in the greatest number of deaths worldwide. Epigenetic changes induced by pregnancy related complication in umbilical cord blood may appear as a result of dysfunctional placenta and impaired maternal cardiovascular function and may cause later onset of cardiovascular diseases in offspring.

Methods: A prospective case control study was conducted at ESIC medical college, Bangalore in 100 PIH women with gestational age 37 to 40 weeks. The study aimed to find the correlation of cord blood uric acid, lipid profile and HOMA -IR in gestational hypertension mothers.

Results: We found a strong positive correlation between uric acid and total cholesterol in cases and a moderate positive correlation among uric acid and LDL in cases whereas a strong negative correlation among uric acid and HDL. In addition, mean HOMA-IR value was statistically significant in cases.

Conclusions: HOMA -IR values are found to be more in newborns of PIH mothers and cord blood uric acid is a good predictor of lipid metabolism and the glucose homeostasis in neonates.

Keywords: Cord blood, Uric acid, Lipid profile, HOMA -IR, Pregnancy induced hypertension

\section{INTRODUCTION}

Cardiovascular diseases (CVD) is a major global health burden that results in the greatest number of deaths worldwide. The WHO estimates that 18.6 million people died from CVD in 2019, accounting for $26.6 \%$ of global mortality. Research shows a strong link between preeclampsia and CVD. Preeclampsia is a pregnancy complication that affects $2-8 \%$ of pregnancies and is a leading cause of maternal and infant morbidity and mortality. ${ }^{1}$

Serum uric acid is a strong predictor of stroke, coronary artery disease, metabolic syndrome definitive role of uric acid is still the subject of much discussion and debate because it is always according with other risk factors such as diet, obesity and dyslipidemia. Uric acid, the final product of purine metabolism in human, is another molecule possessing antioxidant activity. About two-third of antioxidant activity of blood is due to uric acid. ${ }^{2}$

Another study states that serum HDL cholesterol, as a protective factor for CVD risk, is inversely related to uric acid level. It has been lately noted that elevated serum uric acid was a significant predictor of smaller, denser LDL cholesterol and HDL cholesterol particles, which offers a greater atherogenic ability. ${ }^{3}$

Maternal hyperuricemia in normotensive singleton pregnant women is significantly associated with preterm and SGA delivery and the development of neonatal intra ventricular haemorrhage. ${ }^{3}$ Hyperuricaemia in hypertensive pregnancy remains an important finding because it identifies women at increased risk of adverse maternal and 
particularly foetal outcome. ${ }^{4}$ Hence the assessment of cord blood uric acid levels has got wider diagnostic value which is less exploited.

The number of studies harnessing the common predictive parameters like uric acid and lipid profile and exploitation as predictors for the cardiovascular events in their adulthood are less. This study aims at evaluating the relationship of uric acid and the lipid profile parameters like total cholesterol, triglycerides, low density lipoproteins and high density lipoproteins and HOMA-IR that reflects the glucose homeostasis are assessed in cord blood of newborns of pregnancy induced hypertension mothers. Epigenetic changes induced by pregnancy related complication in umbilical cord blood may appear as a result of dysfunctional placenta and impaired maternal cardiovascular function and may cause later onset of cardiovascular diseases in offspring. ${ }^{5}$

\section{Objective}

To evaluate the uric acid, lipid profile levels and HOMAIR in the cord blood of pregnancy induced hypertension. To determine the correlation between the uric acid, lipid profile parameters and HOMA-IR born to PIH mothers.

\section{METHODS}

The case control study was conducted in the Department of Biochemistry in coordination with Department of Pediatrics in ESICMC and PGIMSR, Rajaji nagara, Bangalore during the year 2020 (January to June) and the approval of the study was taken from the ethics and scientific committee of ESIC PGIMSR and model hospital, Bangalore.

\section{Inclusion criteria}

For cases, Gestational age of range 37-40 weeks, only singlet pregnancy with pregnancy induced hypertension diagnosed according to ACOG guidelines where Systolic blood pressure of $140 \mathrm{~mm} \mathrm{Hg}$ or more diastolic blood pressure of $90 \mathrm{mmHg}$ or more on two or more occasions at least 4 hours apart after 20 weeks of gestation in a women with a previously normal blood pressure or Systolic blood pressure of $160 \mathrm{mmHg}$ or more, diastolic blood pressure of $110 \mathrm{mmHg}$ or more.

For controls, gestational age of range 37-40 weeks, only singlet pregnancy without any pregnancy complications.

\section{Exclusion criteria (for both cases and controls)}

All the mothers whose last menstrual period was unknown, multifetal pregnancy, pregnancy complicated by hypothyroidism or any other maternal metabolic disorders and diagnosed any intrauterine infection or congenital anomalies were excluded from the study. For the babies when their APGAR score was less than 7, they were excluded from the study.
The analysis was done with 100 patients with 50 each in two groups, Group H contain hypertensive mothers and Group $\mathrm{N}$ contain normotensive mothers, where all modes of delivery were included.

After considering inclusion and exclusion criteria, under aseptic precaution $3 \mathrm{ml}$ of umbilical (mixed) cord blood is collected in a plain vacutainer, the sample was centrifuged at $5000 \mathrm{rpm}$ for 5 minutes and the serum is stored at 2 to $8^{\circ} \mathrm{C}$.

Estimation of uric acid by enzymatic method is done in Cobas Intergra 400 plus system.

Estimation of lipid profile (total cholesterol by cholestrol oxidase triglycerides by enzymatic method HDL by polymer polyanion, direct measure LDL, VLDL are calculated parameters).

Estimation of glucose by glucose oxidase and peroxidase method.

Estimation of serum insulin by CLIA in Beckman Coulter Access 2.

Calculation of HOMA-IR involved parameters like plasma glucose and Insulin from the formula:

$$
\begin{gathered}
\text { HOMA }-I R=\text { plasma insulin } \times \text { plasma glucose } \\
\div 405
\end{gathered}
$$

Results were entered in the MS excel 2007 and analyzed using descriptive statistics, in the form of frequency, charts and percentage with the help of Statistical package for social sciences (SPSS) version 18.

Following parameters were noted for the newborn: APGAR score, Birth weight and any respiratory distress.

\section{RESULTS}

The mean uric acid level in the cases were $5.64 \pm 0.53$ $\mathrm{mg} / \mathrm{dL}$ as compare to $3.20 \pm 0.610 \mathrm{mg} / \mathrm{dL}$ among controls $(\mathrm{p}<0.05)$. It was observed from the Figure 1A, that there was significant positive correlation between uric acid and total cholesterol in case group, and the same correlation in the control group (Figure 1B). However, the correlation was more strong amongst the cases $(\mathrm{r}=+0.74)$ as compared to controls $(\mathrm{r}=+0.52)$ and the correlation were statistically significant in both groups $(\mathrm{p}<0.05)$. According to the Figure 2, uric acid and HDL showed a strong negative correlation $(\mathrm{r}=-0.86)$ whereas in Figure 3 it depicts Uric acid and LDL having moderate positive correlation $(\mathrm{r}=+0.68)$ amongst cases. The mean HOMAIR levels in cases were 5.02 \pm 0.55 (Figure 2) and in controls were $1.65 \pm 0.21$ and the mean differences were statistically significant $(\mathrm{p}<0.05)$. 
The present study also observed that total cholesterol, triglycerides, LDL levels were high in low birth weight newborns.

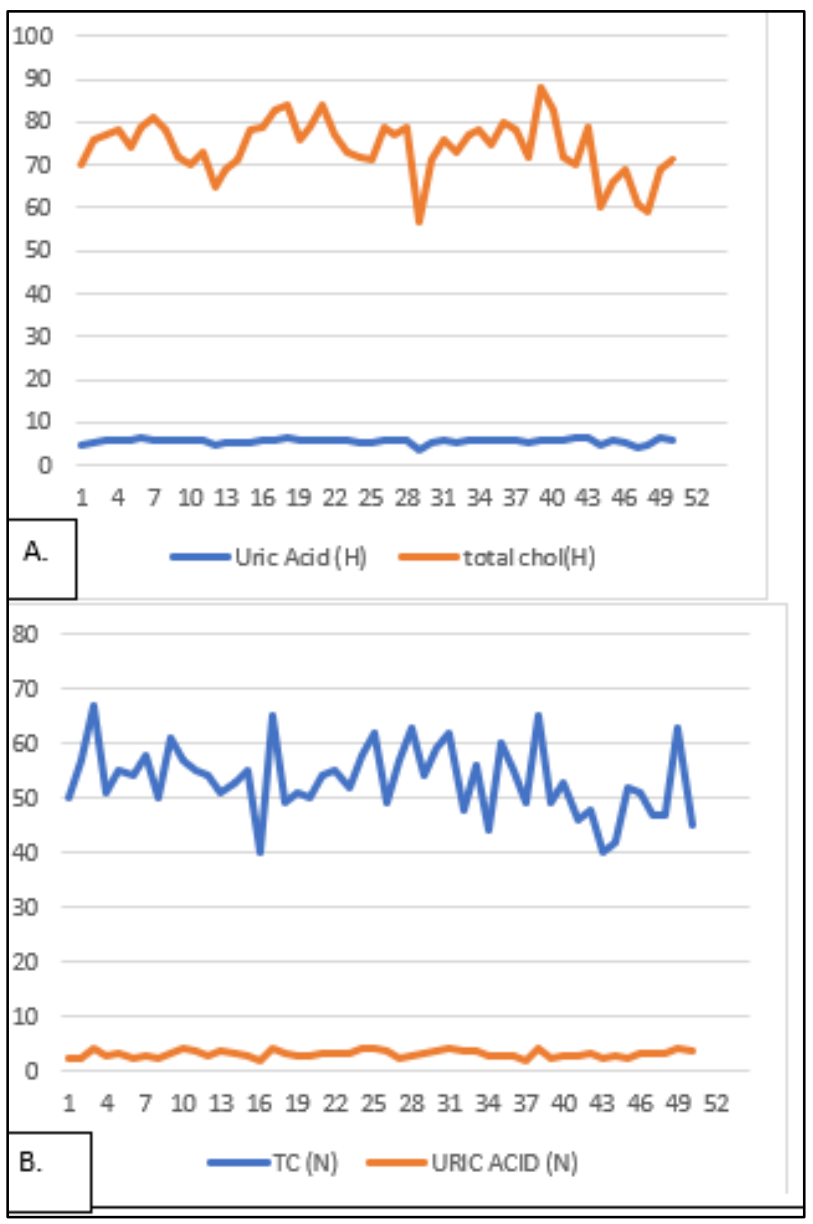

Figure 1: (A) Relationship between cord blood uric acid and total cholesterol in cases (group N). (B)

Relationship between uric acid and total cholesterol in control (Group N).

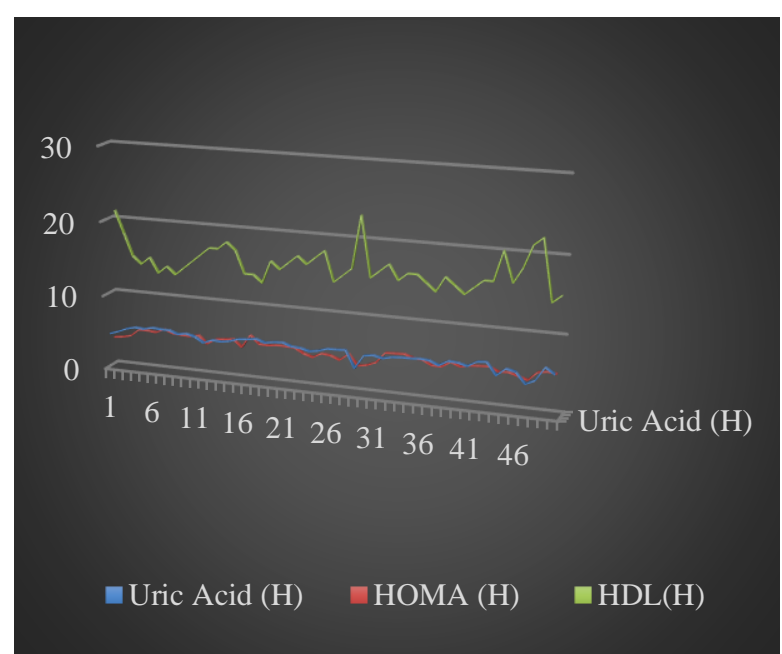

Figure 2: Relationship between uric acid, HDL, HOMA-IR in cord blood of cases.

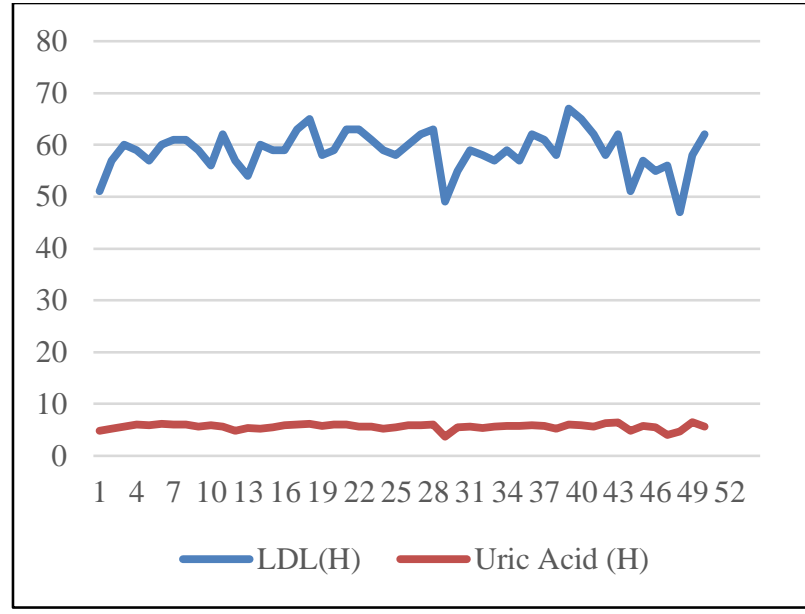

Figure 3: Relationship between cord blood uric acid and $\mathrm{LDL}$ in cases.

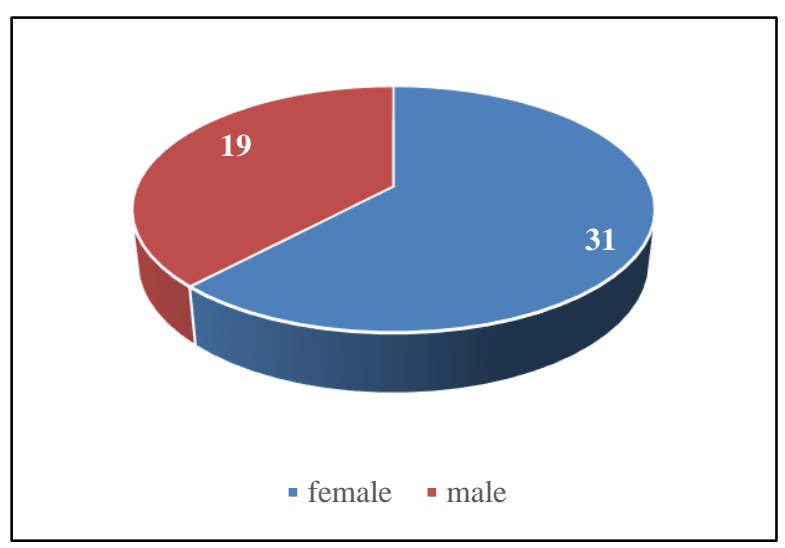

Figure 4: Gender distribution among the cases: Group H.

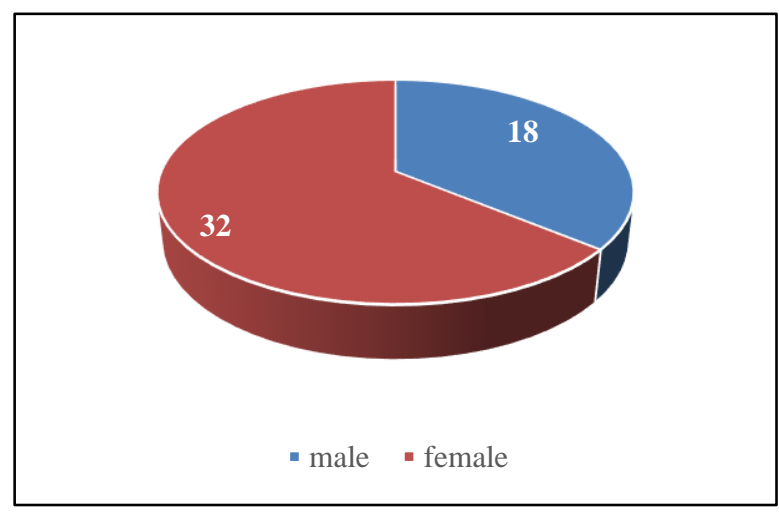

Figure 5: Gender distribution among the controlsGroup N.

The birth weight distribution groupwise (Figure 7), firstly normal birth weight group were $90 \%$ and $86 \%$ of cases and controls respectively and secondly for low birth weight were $10 \%$ and $14 \%$ of cases and controls respectively in the present study. 


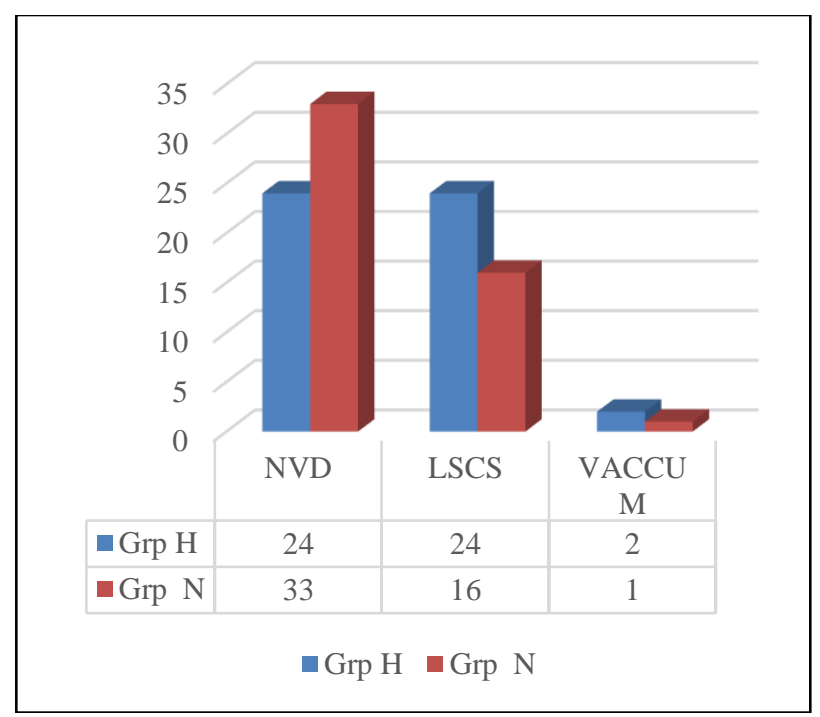

Grp H: Group H (cases), Grp N: Group N (controls), NVD: Normal vaginal delivery, LSCS: Caesarean section, vaccum : Vaccum assisted delivery

Figure 6: Distribution of mode of delivery in both the groups.

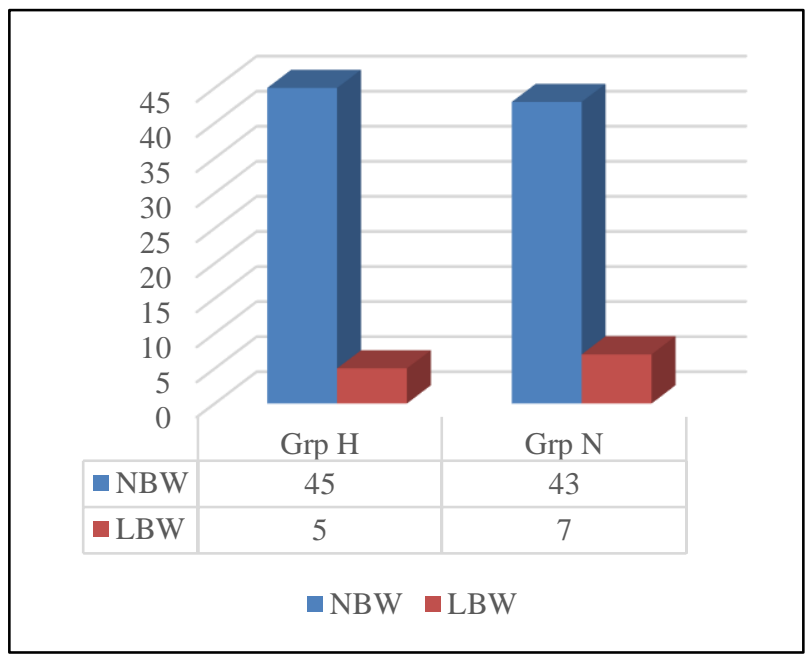

NBW: Normal birth weight (>2500 grams), LBW: low birth weight $(1500-2500$ grams $)$

Figure 7: Birth weight distribution in both groups.

Table 1: Distribution of birth weight in both the groups in the present study.

\begin{tabular}{|lll|}
\hline $\begin{array}{l}\text { Birth weight ranges } \\
\text { (grams) }\end{array}$ & $\begin{array}{l}\text { Group } \\
\text { H }\end{array}$ & Group N \\
\hline $\mathbf{1 5 0 0}-\mathbf{2 5 0 0}$ & 5 & 7 \\
\hline $\mathbf{2 5 0 0}-\mathbf{3 0 0 0}$ & 19 & 29 \\
\hline $\mathbf{3 0 0 0}-\mathbf{3 5 0 0}$ & 21 & 12 \\
\hline $\mathbf{3 5 0 0}-\mathbf{4 0 0 0}$ & 5 & 2 \\
\hline
\end{tabular}

There was no correlation found between birth weight and HOMA IR. The number of babies in the weight range of more than 3000 grams were found to be more in case group than control group which was not statistically significant
(Table 1). Additionally, Figure 4, 5 depicts the gender distribution between the case and control groups and it was found no difference in lipid profile values between male and female newborns. The mode of delivery distribution has been depicted in Figure 6 where it was 48\%, 48\% and $2 \%$ of cases and for controls it was $66 \%, 32 \%$ and $2 \%$ of normal vaginal delivery, caesarean section and Vacuum assisted vaginal delivery respectively.

\section{DISCUSSION}

Studies have proved insulin regulates lipid metabolism and carbohydrates also, where insulin resistance decreases the sensitivity of insulin to adipocytes, myocytes and hepatocytes which leads to increased glucose uptake, weakening the antilipolytic ability that still worsens the situation of hyperinsulinemia. Moreover, it leads to release of more and more free fatty acid into blood stream leading to hyperglycemia with hyperinsulinemia and altered lipid metabolism. ${ }^{6}$

The results of present study confirmed the findings of Kaur et al studies that total cholesterol, triglycerides, LDL were more than mean reference ranges in hypertensive group than the control group and HDL was less in the hypertensive group. ${ }^{7}$ The present study also confirmed the findings of Rodie et al studies reporting that triglycerides and total cholesterol were higher in cord blood of preeclamptic pregnant women, suggesting upregulation of placental transportation mechanism. ${ }^{8}$

Another important factor that was discussed and studied in the present study is birthweight. Birthweight was compared with the lipid profile parameters and the HOMA-IR. It was observed in the present study that babies with birth weight more than 3000 grams were in the case groups which was in line with the Mohammed et al studies, that total cholesterol levels were significantly higher in low birth weight and high birth weight babies which leads to the understanding that low birth wight babies had deranged lipid metabolism which alters the body metabolism reflected in the adulthood as cardiometabolic events is in line with the Barker's Hypothesis. ${ }^{9}$

This study was also in line with Wang et al, who said that the fat accumulation pattern was influenced by cord blood glucose, lipid profile and insulin where the current study shows a predictive value of uric acid and similar parameters in the newborns of pregnancy induced hypertension with the dyslipidemia and cardiovascular events in the adulthood. ${ }^{10}$ The further scope of study is to have an extended observation of the same newborn till the adolescent and adulthood to study the cardio metabolic events.

\section{Limitations}

Small sample size of case and control groups which could be a source of bias. Secondly, the cause effect relationship could not be established. 


\section{CONCLUSION}

The mean reference range of HOMA -IR values were found to be more in newborns of PIH mothers which could be a possible reason for even non-diabetic women giving birth to term neonates. In addition, cord blood uric acid is a good predictor of lipid metabolism and the glucose homeostasis in neonates.

\section{ACKNOWLEDGEMENTS}

Dr. K. Pratibha, Professor and Head, Department of Biochemistry, ESIC MC PGIMSR, Rajaji nagar, Bangalore. Dr. Simant Baliarsingh, Professor, Department of Biochemistry, ESIC MC PGIMSR, Rajaji nagar, Bangalore. Dr.Nanda.K, Associate Professor, Department of Biochemistry, ESIC MC PGIMSR, Rajaji nagar, Bangalore.

Funding: No funding sources Conflict of interest: None declared

Ethical approval: The study was approved by the Institutional Ethics Committee

\section{REFERENCES}

1. World Health Organisation, Global report on Diabetes. Switzerland (Geneva). WHO. 2016. Available at: http://www.who.int. Accessed on 02 June 2021.

2. Amini E, Sheikh M, Hantoushzadeh S, Shariat M, Abdollahi A. Maternal hyperuricemia in normotensive singleton pregnancy, a prenatal finding with continuous perinatal and postnatal effects, a prospective cohort study. Biomed Central. 2014;14:104.

3. Ali N, Rahman S, Islam S, Haque T, Molla NH. The relationship between serum uric acid and lipid profile in Bangladeshi adults. BioMed Central. 2019;19:42.
4. Escendero C, Bertoglia P, Munoz F, Roberts JM. Uric acid and purine plasma levels as plausible markers for placental dysfunction in reeclampsia. 2013;14(7):895902.

5. Hronadnikova I, Kotlabova K, Ivankova K, Vedmetskaya Y, Krofta L. Profiling of cardiovascular and cerebrovascular diseases associated microRNA expression in umbilical cord blood in gestational hypertension, preeclampsia and fetal growth restriction. International Journal of Cardiology. 2017;249:402-9.

6. Kenchappa Y, Bechera N. Assay of neonatal cord blood lipid lipid level and its correlation with neonatal gestational age, gender and birth weight: a single centre experience. International Journal Contemporary Pediatrics. 2016;3:12-4.

7. Kaur K, Thapar K, Malhotra P. Cord blood lipid profile comparison of newborns of hypertensive mothers. Indian Journal of Contemporary Pediatrics. 2020;7(5):1038-42.

8. Rodie VA, Caslake MJ, Stewart F, Sattar N, Ramsay JE, Greer IA et al. Fetal cord plasma lipoprotein status in uncomplicated human preganancies and in pregnancies complicated by Preeclampsia and Intrauterine growth restriction. Atheroscler. 2004;176(1):181-7.

9. Aketayeb SMH, Dehdashtian M, Aminzadeh M, Moghaddam ARE, Mortazavi M. Correlation between umbilical cord blood lipid profile and neonatal birth weight. Pediatria Polska. 2013;88:521-5.

10. Wang J, Shen S, Price MJ, Lu J, Sumilo D. Glucose, Insulin and Lipids in Cord Blood of neonates and their association with birthweight: Differential metabolic risk of Large for gestational age and smaller for gestational age babies. The Journal of Pediatrics. 2020;1-9.

Cite this article as: Priyadharshini R, Vijayakumari V. Cord blood levels of uric acid, lipid profile and HOMA-IR in pregnancy induced hypertension. Int J Reprod Contracept Obstet Gynecol 2021;10:4443-7. 